\title{
Erratum to: 2016 updated MASCC/ESMO consensus recommendations: Prevention of nausea and vomiting following moderately emetogenic chemotherapy
}

\author{
Fausto Roila ${ }^{1}$ - David Warr ${ }^{2}$ Paul J. Hesketh ${ }^{3}$ - Richard Gralla ${ }^{4}$ - Jorn Herrstedt ${ }^{5}$. \\ Karin Jordan $^{6} \cdot$ Matti Aapro $^{7} \cdot$ Enzo Ballatori $^{8} \cdot$ Bernardo Rapoport $^{9}$
}

Published online: 14 September 2016

(C) Springer-Verlag Berlin Heidelberg 2016

Erratum to: Support Care Cancer

DOI 10.1007/s00520-016-3365-1

Table 1 in the original article is unfortunately incorrect. The correct Table 1 is shown below and the original article was corrected.

The online version of the original article can be found at http:// dx.doi.org/10.1007/s00520-016-3365-1.

Fausto Roila

roila.fausto@libero.it

1 Medical Oncology, Santa Maria Hospital, Via Tristano di Joannuccio 1, 05100 Terni, Italy

2 Cancer Clinical Research Unit, Princess Margaret Cancer Centre, Toronto, Canada

3 Lahey Health Cancer Institute, Burlington, USA

4 Albert Einstein College of Medicine, Jacobi Medical Center, New York, NY, USA

5 Department of Oncology, Odense University, 5000 Odense, Denmark

6 Department of Hematology/Oncology, Martin Luther University, Halle-Wittenberg, Halle, Germany

7 Clinique de Genolier, Multidisciplinary Oncology Institute, Genolier, Switzerland

8 Spinetoli, Italy

9 Medical Oncology Centre of Rosebank, 129 Oxford Road, Johannesburg, South Africa 
Table 1 Recommendations for moderately emetogenic chemotherapy

\begin{tabular}{|c|c|c|c|c|}
\hline \multirow[t]{2}{*}{ Chemotherapy } & \multicolumn{2}{|c|}{ Recommendations } & \multirow{2}{*}{$\begin{array}{l}\text { MASCC level of scientific } \\
\text { confidence/level of consensus }\end{array}$} & \multirow{2}{*}{$\begin{array}{l}\text { ESMO level of evidence } \\
\text { grade of recommendation }\end{array}$} \\
\hline & Old & New & & \\
\hline \multicolumn{5}{|l|}{ MEC } \\
\hline Acute & Palo + Dex & $5 \mathrm{HT} 3+\mathrm{Dex}$ & Moderate / moderate & II / B \\
\hline \multirow[t]{2}{*}{ Delayed } & $\operatorname{Dex}^{\mathrm{a}}$ & $\operatorname{Dex}^{\mathrm{a}}$ & Low / moderate & III / C \\
\hline & & None $^{\mathrm{b}}$ & No confidence possible / high & $\mathrm{IV} / \mathrm{D}$ \\
\hline \multicolumn{5}{|c|}{ CARBOPLATIN } \\
\hline Acute & Palo + Dex & $\mathrm{NK} 1+5-\mathrm{HT} 3+\mathrm{DEX}$ & Moderate / moderate & II / B \\
\hline Delayed & $\operatorname{Dex}^{\mathrm{a}}$ & Apre $^{\mathrm{c}}$ & Moderate / moderate & II / B \\
\hline
\end{tabular}

MEC moderately emetogenic chemotherapy, Palo palonosetron, Dex dexamethasone, Apre aprepitant

a only in patients with known potential for delayed emesis

$\mathrm{b}$ the steroid can be considered only in patients receiving MEC with known potential for delayed emesis

${ }^{\mathrm{c}}$ only in patients receiving aprepitant $125 \mathrm{mg}$ on day 1 